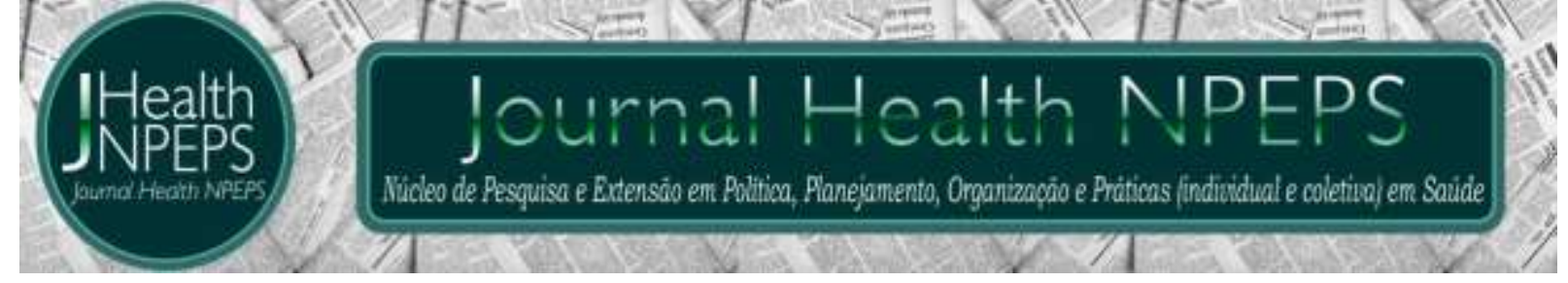

http://dx.doi.org/10.30681/252610103290

ARTIGO ORIGINAL

\title{
Influencia del apoyo social percibido para el sexo seguro en la resiliencia sexual
}

\author{
Influence of social support perceived for safe sex in sexual resilience
}

Influência do apoio social percebido para o sexo seguro na resiliência sexual

\author{
Sandra Paloma Esparza-Dávila ${ }^{1}$, María Guadalupe Moreno-Monsiváis²
}

\section{RESUMEN}

Objetivo: determinar la influencia del apoyo social percibido (apoyo de familia, amigos, pareja y proveedores de salud) para el sexo seguro en la resiliencia sexual. Método: estudio descriptivo correlacional, participaron 202 adolescentes mexicanos (136 mujeres y 66 hombres), entre 14 y 17 años $(M=15 ; D E=0.663)$. Se aplicó una cédula de datos, la Escala Provisión Social y Escala de Resiliencia, ambas para sexo seguro. Resultados: los resultados indican que el apoyo de la familia, pareja y proveedores de salud influyen significativamente en la resiliencia sexual del adolescente, explicando el $33 \%$ de la varianza $(F[4,197]=25.91, p<0.001)$. Con respecto al apoyo de los amigos se encontró una influencia negativa y no significativa hacia la resiliencia sexual. Conclusión: estos resultados muestran que el apoyo de la familia, pareja y proveedores de salud son de los principales recursos con los que cuenta el adolescente para afrontar diversos cambios y situaciones relacionados a la sexualidad, lo que le permitirá tener una conducta resiliente.

Descriptores: Resiliencia Psicológica; Conducta Sexual; Apoyo Social; Adolescente.

\section{ABSTRACT}

Objective: to determine the influence of perceived social support (support from family, friends, partners and health providers) for safe sex in sexual resilience. Method: a correlational descriptive study involving 202 Mexican adolescents (136 women and 66 men), between 14 and 17 years old $(M=15, S D=0.663)$. A data card was applied, Social Provision Scale for Safe Sex and Scale of Resilience for Safe Sex. Results: the results indicate that the support of the family, couple and health providers significantly influence the adolescent's sexual resilience, which explains the

\footnotetext{
${ }^{1}$ Enfermera. Doctorado. Coordinador de Investigación y maestra de tiempo completo. Universidad de Monterrey. Monterrey, México. E-mail: mce.esparzasp@outlook.com ORCID ID: https://orcid.org/0000-0002-3482-5057 Autor principal - Endereço para correspondência: Av. Ignacio Morones Prieto 4500, 66238 San Pedro Garza García, N.L., México.

${ }^{2}$ Enfermera. Doctorado. Maestra de Tiempo Completo de la Facultad de Enfermería de la Universidad Autónoma de Nuevo León. Universidad Autónoma de Nuevo León. Monterrey, Nuevo León, México. E-mail: mgmoreno@hotmail.com ORCID ID: https://orcid.org/0000-0002-7152-0244
}

Este artigo está licenciado sob forma de uma licença Creative Commons Atribuição 4.0 Internacional, que permite uso irrestrito, distribuição e reprodução em qualquer meio, desde que a publicação original seja corretamente citada. 
$33 \%$ variance $(F[4,197]=25.91, p<0.001)$. With respect to the support of friends, $a$ negative and not significant influence towards sexual resilience was found. Conclusion: these results show that the support of the family, couple and health providers are the main resources available to the adolescent to face various changes and situations related to sexuality, which will allow him to have a resilient behavior.

Descriptors: Resilience, Psychological; Sexual Behavior; Social Support; Adolescent.

\section{RESUMO}

Objetivo: determinar a influência do apoio social percebido (apoio da família, amigos, parceiros e provedores de saúde) para o sexo seguro na resiliência sexual. Método: estudo descritivo correlacional, envolvendo 202 adolescentes mexicanos (136 mulheres e 66 homens), entre 14 e 17 anos de idade $(M=15, D P=0,663)$. Foi aplicado um cartão de dados, Escala de Provisão Social para Sexo Seguro e Escala de Resiliência para Sexo Seguro. Resultados: os resultados indicam que os grupos de apoio da família e os prestadores de saúde influenciam significativamente a resistência sexual adolescente, correspondendo a $33 \%$ da variância $(F[4,197]=25,91, p<0,001)$. Com relação ao apoio de amigos, foi encontrada uma influência negativa e não significativa em relação à resiliência sexual. Conclusão: estes resultados mostram que o apoio da familia, cônjuge e prestadores de cuidados de saúde são os principais recursos que o adolescente possui para enfrentar várias mudanças e situações relacionadas com a sexualidade, o que lhe permitirá ter um comportamento resiliente.

Descritores: Resiliência Psicológica; Comportamento Sexual; Apoio Social; Adolescente.

\section{INTRODUCCIÓN}

La Organización Mundial de la Salud (OMS) refiere que los adolescentes están en constantes cambios biológicos, psicológicos y sociales. En esta etapa buscan nuevas experiencias, lo que los lleva a involucrarse en conductas de riesgo sexual como embarazos no deseados, relaciones sexuales sin utilizar el condón, múltiples parejas sexuales y contagio de Infecciones de Transmisión Sexual (ITS) destacando VIH-Sida ${ }^{1}$. La literatura señala que existen factores de protección que pueden ayudar a disminuir estos riesgos, de acuerdo al Modelo de Resiliencia de Adolescentes,

Journal Health NPEPS. 2019 jan-jun; 4(1):80-91. estos factores involucran el nivel individual, familiar y social ${ }^{2}$.

En este sentido, la familia, amigos, pareja y proveedores de salud ${ }^{3}$, son fuentes de apoyo para prevenir los riesgos de comportamientos que sean contrarios a la salud 4 como lo son las conductas sexuales de riesgo. La familia se considera como la unidad básica de salud que mediante una autoridad afectiva y responsable, puede ser un elemento protector para el adolescente ${ }^{5}, 6$. Por otra parte, se encuentran los amigos, en los cuales el adolescente suele encontrar consejos y respuesta a sus dudas de alguna relación de noviazgo o aspectos de sexualidad. Diversos investigadores mencionan que 
estas inquietudes dependen más del comportamiento del grupo de amigos que del desarrollo biológico del adolescenter.

De manera similar, otra persona de la que percibe apoyo es la pareja la cual es importante en la toma de decisiones respecto a la sexualidad ${ }^{8}$. Sin embargo, en relación a los amigos y pareja, se ha reportado que si bien, pueden ser una fuente de apoyo para una conducta sexual segura, también pueden tener un efecto negativo $y$ convertirse en factores de riesgo ${ }^{7}$.

Por último, los proveedores de salud al ser quienes establecen estrategias de promoción de salud y conocimientos que ayudan a fomentar estilos de vida saludables, puede ser que brinden a los adolescentes las herramientas necesarias para que tengan conductas sexuales seguras ${ }^{9}$. Cuando el apoyo social se brinda de manera adecuada, el adolescente puede llegar a tener conductas con recursos suficientes que le permitan decidir y analizar situaciones de riesgo para el sexo seguro, si el adolescente logra esas conductas de protección se logra la resiliencia sexual ${ }^{2}$.

De acuerdo a lo anterior el objetivo del presente estudio es determinar la influencia del apoyo social percibido (apoyo de familia, amigos, pareja y proveedores de salud) para el sexo seguro en la resiliencia sexual.

\section{MÉTODO}

El diseño del estudio fue de tipo descriptivo correlacional en adolescentes de 14 a 17 años de una preparatoria de una institución pública del área metropolitana de Monterrey, Nuevo León, México. Se utilizó un muestreo aleatorio simple, la muestra fue de 202 adolescentes, estimada para una prueba de regresión lineal múltiple con un coeficiente de determinación de 0.09, un nivel de confianza del $95 \%$ y una potencia del 90\%. Los criterios de inclusión fueron adolescentes de 14 a 17 años de edad y de exclusión fueron adolescentes que estuvieran en unión libre o casados.

Para medir los datos sociodemográficos se utilizó una cédula de datos que incluía preguntas como: edad, sexo, semestre y conocer si el adolescente se encontraba en una relación de noviazgo o estaba soltero. Por otra parte para medir la variable de apoyo social percibido para sexo seguro, se utilizó la Escala Provisión Social para Sexo Seguro ${ }^{10}$ la cual contiene 78 reactivos divididos en 4 sub escalas 
(apoyo de la familia, pareja, amigos y proveedores de salud). Las opciones de respuesta son tipo Likert de 1 a 5 , donde 1 es totalmente en desacuerdo, 2 en desacuerdo, $3 \mathrm{Ni}$ de acuerdo ni en desacuerdo, 4 de acuerdo y 5 totalmente de acuerdo. Una mayor puntuación indica mayor percepción de apoyo. La escala ha reportado un Alfa de Cronbach de $0.94^{11}$.

Respecto a la variable de resiliencia sexual en el adolescente se utilizó la Escala de Resiliencia para Sexo Seguro $^{12}$, la cual está integrada por 22 ítems, cuenta con dos sub escalas competencia personal y aceptación de sí mismo y de la vida, que en conjunto miden la resiliencia sexual. Las opciones de respuestas son tipo Likert que van de 1 a 5 puntos, 1 significa muy en desacuerdo, 2 en desacuerdo, 3 indeciso, 4 de acuerdo y 5 muy de acuerdo. La puntuación oscila de 22 a 110 puntos, a mayor puntaje se indica un alto nivel de resiliencia sexual. Ha reportado un Alfa de Cronbach de 0.9411.

Para la recolección de la información, se solicitó la aprobación de los Comités de Investigación y Ética en Investigación de la Facultad de Enfermería de la Universidad Autónoma de Nuevo León (FAEN-M-1067), así como de los directivos de la institución pública donde se realizó el estudio, apegándose a lo descrito en la Ley General de Salud en Materia de Investigación para la Salud ${ }^{13}$.

Una vez obtenidos los permisos se procedió a seleccionar a los adolescentes de manera aleatoria mediante las listas de cada grupo. Se verificó que los estudiantes seleccionados de manera aleatoria no estuvieran casados o vivieran en unión libre; a todos aquellos que reunieron el requisito se les invitó a participar en el estudio, se les explicó el objetivo y si aceptaban participar se les entregaba un consentimiento informado para que sus padres autorizaran su participación, así como un asentimiento informado para que el adolescente lo firmara. Al regresar estos documentos, se les pidió un correo electrónico para enviarles un link del portal Survey Monkey y pudieran contestar los instrumentos. En todo momento se les garantizó que la información proporcionada era anónima y confidencial y que solo sería revisada por el equipo de investigación.

Para el análisis estadístico se utilizó el paquete estadístico SPSS versión 21. El análisis consistió en estadística descriptiva: frecuencias, porcentajes y medidas de tendencia central y dispersión. Para el análisis 
inferencial se aplicó el coeficiente de correlación de Spearman para examinar la relación del Apoyo Social Percibido para Sexo Seguro y la Resiliencia Sexual, así como una prueba de regresión lineal para determinar la influencia del Apoyo Social Percibido para Sexo Seguro en la Resiliencia Sexual en adolescentes.

\section{RESULTADOS}

La muestra fue de 202 adolescentes de una preparatoria del área metropolitana de Monterrey, Nuevo León, México. El estudio se conformó por adolescentes de 14 a 17 años $(\bar{X}=15$ años, $D E=0.663)$, predominó el género femenino (67.3\%). En relación a la situación sentimental el $70.3 \%$ no tenía pareja. En la Tabla 1 se muestra que el apoyo que perciben los adolescentes es alto ya que en todas las subescalas: Familia, Pareja, Amigos y Proveedores de Salud la media estuvo por encima del punto intermedio.

Tabla 1 - Apoyo Social Percibido para Sexo Seguro por los adolescentes.

$\begin{array}{llll}\text { Variable } & \text { Media } & \text { Mdn } & \text { DE } \\ \text { Apoyo Social Percibido } & 3.79 & 3.80 & 0.44 \\ \text { Familia } & 3.73 & 3.76 & 0.61 \\ \text { Pareja } & 3.88 & 3.90 & 0.66 \\ \text { Amigos } & 3.67 & 3.66 & 0.54 \\ \text { Proveedores de Salud } & 3.87 & 3.88 & 0.59\end{array}$

Media=Promedio aritmético; Mdn = Mediana: $D E=$ Desviación estándar

Journal Health NPEPS. 2019 jan-jun; 4(1):80-91.
En la Tabla 2 se muestran los resultados de la Resiliencia Sexual (Competencia Personal y Aceptación de sí mismo y de la vida), como puede observarse la media se ubicó por encima del punto intermedio, lo que indica que los adolescentes se perciben resilientes sexualmente.

Tabla 2 - Resiliencia Sexual percibida por los adolescentes.

\begin{tabular}{lccc}
\hline Variable & Media & Mdn & $D E$ \\
Resiliencia Sexual & 3.94 & 4.00 & 0.54 \\
Competencia personal & 3.91 & 4.00 & 0.62 \\
$\begin{array}{l}\text { Aceptación de sí mismo y } \\
\text { de la vida }\end{array}$ & 4.09 & 4.25 & 0.82 \\
\hline $\begin{array}{l}\text { Media=Promedio aritmético; } \\
\text { DE = Desviación estándar. }\end{array}$ & & & \\
\hline
\end{tabular}

En relación al objetivo que consistió en examinar la relación que existe entre el Apoyo Social Percibido para Sexo Seguro (Apoyo de la Familia, Pareja, Amigos y Proveedores de Salud) y la Resiliencia Sexual (Competencia Personal y Aceptación de sí mismo y de la vida) de los adolescentes, mediante la prueba de correlación de Spearman se encontró una correlación significativa entre ambas variables $\left(r_{s}=0.548, p<\right.$ 0.01) (Tabla 3).

Al analizar la relación entre las subescalas de apoyo social percibido para sexo seguro con la Resiliencia Sexual se encontró una correlación positiva con las subescalas de Apoyo de la Familia $\left(r_{s}=0.360, p<.01\right)$, Apoyo de la Pareja $\left(r_{s}=0.468, p<0.01\right)$, Apoyo de 
los Amigos $\left(r_{s}=0.319, p<0.01\right)$ y Apoyo de los Proveedores de Salud $\left(r_{s}=0.493, p\right.$ < 0.01) (Tabla 3).

Tabla 3 - Correlación entre las subescalas que integran el Apoyo Social Percibido para Sexo Seguro con Resiliencia Sexual.

Variable

1.Apoyo 1

Social

Percib.

2.Fam.

\subsection{5}

3.Pareja

$1^{* *}$

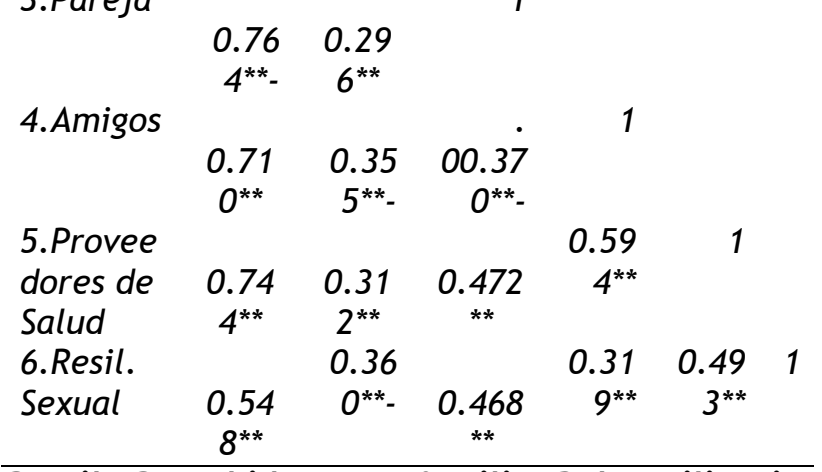

Percib: Percebido; Fam: familia; Rel: resiliencia Correlación de Spearman $\left(r_{s}\right) . \quad{ }^{*} p<0.05$. ${ }^{* *} p<.01$. $n=202 *$.

Para determinar la influencia del Apoyo Social Percibido (Apoyo de Familia, Amigos, Pareja y Proveedores de Salud) para el Sexo Seguro en la Resiliencia Sexual (Competencia Personal y Aceptación de sí mismo y de la vida) se llevó a cabo una prueba de Regresión Lineal.

Se determinó como variable dependiente la Resiliencia Sexual y la variable independiente el Apoyo Social Percibido para Sexo Seguro. LoS resultados mostraron que la variable de estudio analizada de manera global explica el $30 \%$ de la varianza para la Journal Health NPEPS. 2019 jan-jun; 4(1):80-91.
Resiliencia Sexual $\left(F_{[1,200]}=90.90, p<\right.$ 0.001), lo cual indica que el Apoyo Social Percibido para Sexo Seguro influye significativamente en la Resiliencia Sexual.

Posteriormente se analizaron las subescalas de la variable independiente Apoyo de la Familia, Pareja, Amigos y Proveedores de Salud con respecto a la variable dependiente Resiliencia Sexual. Los resultados indican que las subescalas explican el 33\% de la varianza para la Resiliencia Sexual $\left(F_{[4,197]}=25.91, p\right.$ <0.001) por lo que el Apoyo de la Familia, Pareja y Proveedores de Salud influyen significativamente en la Resiliencia Sexual del adolescente.

Con respecto al Apoyo de los Amigos se encontró una influencia negativa y no significativa hacia la Resiliencia Sexual (Tabla 4).

Tabla 4 - Prueba de Regresión Lineal Múltiple de las subescalas que integran el Apoyo Social Percibido para Sexo Seguro con Resiliencia Sexual.

\begin{tabular}{|c|c|c|c|c|c|}
\hline Subescalas & $F$ & $d f$ & $\begin{array}{c}\text { Error } \\
\text { Estándar }\end{array}$ & $\begin{array}{c}\text { Coeficientes } \\
\text { estandariza } \\
\text { dos } \\
\text { Beta }\end{array}$ & $p$ \\
\hline Familia & 25.91 & 4 & 0.056 & 0.181 & $\begin{array}{r}0.0 \\
04\end{array}$ \\
\hline Pareja & 25.91 & 4 & 0.055 & 0.297 & $\begin{array}{c}0.0 \\
01\end{array}$ \\
\hline Amigos & 25.91 & 4 & 0.075 & -0.055 & $\begin{array}{r}0.4 \\
54\end{array}$ \\
\hline $\begin{array}{l}\text { Proveedores } \\
\text { de Salud }\end{array}$ & 25.91 & 4 & 0.070 & 0.327 & $\begin{array}{c}0.0 \\
01\end{array}$ \\
\hline
\end{tabular}

$F=$ Distribución $F$. $d f=$ Grados de Libertad. Variable dependiente=resiliencia sexual. $R^{2}$ ajust. $=.33$ 


\section{DISCUSIÓN}

La adolescencia es una etapa crítica del desarrollo humano, se caracteriza por cambios físicos y desafíos que se presentan de manera evolutiva, parte de estos cambios generan que los adolescentes puedan incurrir en prácticas sexuales de riesgo. Hoy en día las conductas sexuales de los adolescentes es un tema de suma importancia por las implicaciones que tienen en la salud, diversas investigaciones refieren datos alarmantes sobre las consecuencias de estas conductas. Con frecuencia los adolescentes, tienen pensamientos erróneos sobre la sexualidad, llegan a pensar que el tener relaciones sexuales no es de riesgo, por lo que no son conscientes de las consecuencias de sus acciones. Ante esto, es necesario consolidar los recursos con los que cuenta el adolescente como lo es el apoyo social, para afrontar los diversos cambios que los pueden hacer vulnerables a riegos de tipo sexual.

En la presente investigación se encontró una relación positiva entre el Apoyo Social Percibido (Familia, Amigos, Pareja y Proveedores de Salud) para Sexo Seguro y la Resiliencia Sexual (Competencia Personal y Aceptación de sí mismo y de la vida) del adolescente, hallazgo similar a lo reportado en algunas investigaciones 14, 20. Por una parte el apoyo social al hacer referencia al conjunto de aportes que se reciben de las relaciones establecidas con otros, es un factor determinante en la salud de los adolescentes ${ }^{21}$.

Las estructuras de apoyo social como la familia, amigos, pareja y proveedores de salud experimentan cambios que son significativos para los adolescentes, la combinación de atributos del individuo y su ambiente familiar, social y cultural posibilitan la superación de riesgos en las conductas sexuales y generan que el adolescente sea resiliente. La resiliencia sexual favorece conductas seguras, una influencia positiva genera que el adolescente tenga comportamientos responsables que les ayudan a desarrollar la capacidad de responder de manera crítica y resolutiva situaciones de riesgo sexual ${ }^{22}$ estimulando la necesidad de optar por mejores condiciones de salud.

Otro de los aspectos analizados fue el apoyo que proviene de la familia el cual se relacionó con la resiliencia sexual, resultado que fue similar a lo reportado en otras investigaciones ${ }^{5,15,16}$. Al respecto, algunos estudios muestran 
que la percepción de apoyo que el adolescente percibe de la familia, es un factor protector frente a conductas de riesgo sexual, como lo es el inicio temprano de actividad sexual ${ }^{23}$. La familia se considera como el grupo de pertenencia central de los adolescentes, es la referencia que marca la interacción social, los padres son la fuente de protección del bienestar personal del adolescente para evitar riesgos en la salud. En lo que respecta a la sexualidad, cuanto más conocedor es un padre sobre temas de sexualidad, más confianza siente para discutir esos temas con sus hijos adolescentes y esto contribuye positivamente a lograr la resiliencia sexual ${ }^{5,15,16}$.

Otro de los que son partícipes en el apoyo social son los amigos y la pareja, en el presente estudio tanto los amigos y pareja mostró relación con la resiliencia sexual, sin embargo el apoyo de los amigos fue negativo, lo cual concuerda con algunos autores ${ }^{17}$, ${ }^{18}$. El apoyo social por parte de los amigos ha mostrado ser un factor de riesgo para la iniciación y mantención de conductas sexuales de riesgo, parte de la explicación de este aspecto es que los amigos son la fuente de información y muchas de las veces, la información con la que cuentan sobre temas de sexualidad, no es la correcta. Referente al apoyo de la pareja y de acuerdo a la literatura, ésta se considera como un medio que provee información y no como una persona que ayude al adolescente a ser resiliente ${ }^{17,18}$.

Por último se encontró que el apoyo de los proveedores de salud se relacionó con la resiliencia sexual, hallazgo similar a lo reportado por diversos autores ${ }^{19,} 20$, quienes refieren que el papel que ejerce enfermería con los adolescentes respecto a información de sexualidad, ayuda a que los adolescentes puedan tener conductas resilientes y practicar sexo seguro. En este sentido, al ser el apoyo social un factor protector, genera que los adolescentes puedan tomar decisiones asertivas sobre los cambios que se presenten en sus conductas sexuales.

\section{CONCLUSIÓN}

Ante los hallazgos del estudio se puede concluir que el apoyo de la familia, pareja y proveedores de salud son de los principales recursos con los que cuenta el adolescente para afrontar diversos cambios y situaciones relacionados a la sexualidad, lo que le permitirá tener una conducta resiliente. La familia, pareja y proveedores de 
salud deben de contar con información de sexualidad correcta, además, es importante fortalecer las estrategias que llevan a cabo para trasmitir información de sexualidad a los adolescentes de manera que les permitan ser autoeficaces y lograr que el adolescente sea resiliente.

Cuando se trabaja con los adolescentes, es fundamental tomar en cuenta la calidad de la información que reciben de los diferentes miembros de su red social, este es un aspecto relevante que debe ser analizado por los proveedores de salud para plantear estrategias de prevención que respondan a las necesidades reales de información de los adolescentes.

Una de las limitaciones identificadas en el presente estudio se atribuye al diseño de la investigación el cual no permite identificar causalidad, por otro lado los resultados no pueden ser generalizados a población adolescente en general, solo se limita a población con características similares. Por otra parte, en México, hablar y tratar temas de sexualidad es complicado debido a la cultura que impera y los tabúes que existen en la población. Para próximas investigaciones se sugiere analizar por separado las poblaciones de adolescentes cuya situación sentimental sea noviazgo o sin pareja, así como investigar si la resiliencia sexual varía de acuerdo al género del adolescente y si el apoyo familiar es distinto de acuerdo a la estructura familiar.

\section{REFERENCIAS}

1. Organización Mundial de la Salud. Infecciones de transmisión sexual. 2016. [acceso 10 de enero 2018]. Disponible en: http://www.who.int/es/newsroom/fact-sheets/detail/sexuallytransmitted-infections-(stis)

2. Haase JE. The adolescent resilience model as a guide to interventions. J Pediatr Oncol Nurs. 2004; 21(5):289-99.

3. Brown SG, Hudson DB, CampbellGrossman C, Kupzyk KA, Yates BC, Hanna KM. Social Support, Parenting Competence, and Parenting Satisfaction Among Adolescent, African American, Mothers. West J Nurs Res. 2018; 40(4):502-19.

4. Páramo MÁ. Factores de Riesgo y Factores de Protección en la Adolescencia : Análisis de Contenido a través de Grupos de 
Discusión. Ter Psicol. 2011; 29(1): 85-95.

5. Baku EA, Agbemafle I, Adanu RMK. Effects of parents training on parents' knowledge and attitudes about adolescent sexuality in Accra Metropolis, Ghana. Reprod Health. 2017; 14(1):101.

6. Dávila SPE, Champion JD, Monsiváis MGM, Tovar M, Arias MLF. Mexican Adolescents' SelfReports of Parental Monitoring and Sexual Communication for Prevention of Sexual Risk Behavior. J Pediatr Nurs. 2017; 35:83-9.

7. Alfonso FL, Figueroa PL. Conductas sexuales de riesgo en adolescentes desde el contexto cubano. Rev cienc med. 2017; 21(2):143-51.

8. De Jesús RD, Cabello MC. Sexualidad y reproducción adolescentes: un estudio sociocultural en un contexto urbano-marginal de Monterrey, Nuevo León, México. Rev sexol soc. 2014; 17(46):14-25.

9. González EA, Molina TG, San Martin JV. Comportamientos sexuales y características personales según orientación sexual en adolescentes chilenos.
Rev Chil Obs Ginecol. 2016; 81(3):202-10.

10. Darbes LA, Lewis MA. HIV-specific social support predicts less sexual risk behavior in gay male couples. Health Psychol. 2005; 24(6):617-622.

11. Castillo-Arcos L, Benavides-Torres R. Modelo de resiliencia sexual en el adolescente: Teoría de rango medio. Aquichan. 2012; 12(2):16982.

12. Heilemann MV, Lee KA, Kury FS. Strengths and vulnerabilities of women of Mexican descent in relation to depressive symptoms. Nurs Res. 2002; 51(3):175-82.

13. Secretaría de Salud. Reglamento de la Ley General de Salud en Materia de Investigación para la Salud. México; 1987 [acceso 10 de enero 2018]. Disponible en: https://archivos. juridicas.unam.m x/www/bjv/libros/5/2292/63.pdf

14. Wright MOD, Masten AS, Narayan AJ. Resilience processes in development: Four waves of research on positive adaptation in the context of adversity. Boston: Springer; 2013.

15. Rivadeneira J, López MA. Escala de Comunicación Familiar: 
validación en población adulta chilena. Acta Colomb Psicol. 2017; 20(2):116-26.

16. Raboteg-Saric $Z$, Sakic $M$. Relations of parenting styles and friendship quality to self-esteem, life satisfaction and happiness in adolescents. Appl Res Qual Life. 2014; 9(3):749-65.

17. Zúñiga $A$, Teva $I$, Bermúdez $M$. Conocimiento y Fuentes de Información sobre las ITS/VIH, Comunicación sobre Sexo y Actitud Hacia el Uso del Preservativo en Adolescentes y Padres/Madres Salvadoreños. Rev Iberoam Diagn Ev. 2017; 3(45):97107.

18. Ruiz-Canela M, López-del Burgo C, Carlos S, Calatrava M, Osorio A, Irala J. Familia, amigos y otras fuentes de información asociadas al inicio de las relaciones sexuales en adolescentes de El Salvador. Rev Panam Salud Pública. 2012; 31(1):54-61.
19. Sáenz NE. Modelo para la promoción del sexo seguro en parejas estables (Tesis de doctorado). Monterrey: Facultad de Enfermería, Universidad Autónoma de Nuevo León; 2014. $260 \mathrm{p}$.

20. Díaz A, Parra S, Aravena V, Barriga O. Validez y confiabilidad de una escala de apoyo social percibido en población adolescente. Enferm Glob. 2015; 14(3):125-136.

21. Organización Mundial de la Salud. Social determinants of health. The solid facts. 2003.

22. Díaz G. Consideraciones teóricas acerca del empoderamiento psicológico en salud sexual de actores sexuales vinculados a niños/as preescolares. Rev Cubana Med Integr. 2011; 27(1):23-32.

23. Díaz C, González M. Conductas problemas en adolescentes en la ciudad de Monterrey, México. Enferm Glob. 2014; 13(1):1-16.

Conflito de interesses: Os autores declaram não haver conflito de interesses.

\section{Participação dos autores:}

- Concepção: Esparza-Dávila SP, Moreno-Monsiváis MG.

- Desenvolvimento: Esparza-Dávila SP, Moreno-Monsiváis MG.

- Redação e revisão: Esparza-Dávila SP, Moreno-Monsiváis MG. 
Como citar este artigo: Esparza-Dávila SP, Moreno-Monsiváis MG. Influencia del apoyo social percibido para el sexo seguro en la resiliencia sexual. J Health NPEPS. 2019; 4(1):80-91.

Submissão: $11 / 01 / 2019$

Aceito: $20 / 05 / 2019$

Publicado: 01/06/2019 